Running Head: Robust and Replicable Science in Infant and Developmental Research

\begin{abstract}
An Overview of Issues in Infant and Developmental Research for the creation of Robust and Replicable Science
\end{abstract}

Pamela E. Davis-Kean and Alexa Ellis University of Michigan

Paper Accepted 7/8/2019: Infant Behavior and Development

Author's Note: We would like to thank members of the Human Development and Quantitative Methods Lab (HDQM) for helpful comments on earlier versions of this draft as well both the reviewers and Editor of the paper. 


\begin{abstract}
In the last few years, the field of psychology has been challenged with a crisis in the rigor and reproducibility of the science. The focus of these issues has primarily been in social, cognitive, and cognitive neuroscience psychology, however, the area of developmental research is not immune to these issues. This paper provides an overview of the "replication crisis" and the choices made by researchers that are often not noted in methods, thus making the replication of studies more difficult. In this review we discuss issues of researcher flexibility in the data design and selection of sample size, collection, and analysis stages of research. In each of these areas we address examples of bias and how developmental researchers can address these issues in their own research.
\end{abstract}




\section{An Overview of Issues in Infant and Developmental Research for the creation of Robust and Replicable Science}

At the 2013 Association of Psychological Science (APS) Annual Convention, two major symposia were delivered that discussed the beginning of what is now termed the "psychological crisis.” The crisis as defined by the speakers was related to problematic data practices (e.g, adjusting $p$-values, hypothesizing after analyzing data, selectively dropping and adding participants to obtain significant $p$-values) and issues of replicability of psychological phenomena (also noted as the "replication crisis"). Even though these issues were starting to appear in published papers (see Simmons, Nelson, \& Simonsohn, 2011) prior to this meeting, this was the first time they were discussed at a major psychological conference. As a result, a call for action was put forward to make psychological science more rigorous and the findings more replicable across all areas of psychology. Most of the examples provided at the meeting were related to the cognitive, social, and neuroscience areas of psychology where issues of small samples (i.e., low power) and lack of multiple comparison corrections are relatively frequent in publications. However, when the examples were presented at the meeting, developmentalists in the room recognized the similarities in their own research practices, and that we were not immune to the issues of research replicability and rigorous practices (see Munafò et al., 2017).

At the APS convention, Jelte Wicherts presented research conducted with Marjan Bakker (Bakker \& Wicherts, 2011) that examined high- and low-impact journals across the area of psychology to determine how often the $p$-value reported in the publication was incorrect. In the developmental area there were four journals that were reviewed for the full year of publication in 2008. For high-impact journals (e.g. Development and Psychopathology), close to 50\% of the papers published in that year had published p-value errors. In the low-impact journals (e.g. 
Journal of Applied Developmental Psychology) the error rate ranged from 54-67\%. Thus, errors in reporting the $p$-value of data were common practices in publications, regardless of journal impact factor, contributing to publication bias in the developmental literature.

In general, most developmental researchers would agree that this type of manipulation of the data for the sake of publishing a significant result is unethical; however, there are other more questionable research practices that are common in the developmental area and particularly those using infants that may also lead to false positive results and challenge the rigor of the research produced. In this paper we will review some of the areas of practice that are most relevant to infancy and early childhood research. These practices can be categorized into three main areas of responsible scientific conduct: statistical power and sample size, protocol flexibility, and analysis flexibility. We will briefly discuss some of the most common concerns relevant to developmental psychology, and then offer recommendations to address these issues and increase the rigor and replicability of research on the early years of life.

\section{Statistical Power and Small Sample Size}

There have been multiple articles written on the issues of statistical power of proposed research designs in the literature (Ioannidis, 2005; Lindsay, 2015; Wicherts et al., 2016). Yet, some of the most common problems in the developmental literature involve trying to ascertain the effect size or range of effect sizes for the phenomena being studied. Due to publication biases and small samples, however, we are in a strange circular situation where we estimate power based on prior research that had inflated effect sizes, which then leads to additional publications with inflated effects. Determining the most accurate effect size is complicated but important for designing robust studies. Thus, how can developmental researchers determine the best estimate? 
For decades, it has been common to refer to Cohen’s suggestions (Sawilowsky, 2009) for effect size magnitude (.20 for small, .50 for medium, and .80 for large effects) when designing a study and determining the sample size needed to adequately find these effects. Unfortunately, these “rule of thumb” estimates are rarely found in psychological research. Many of the psychological phenomena being studied are of modest size at best (less than .25) and thus using Cohen effect sizes may not provide the best estimates of the sample sizes needed for all outcomes examined in infant research (Open Science Collaboration, 2015).

Other strategies for determining effect size for power calculations include seeking out effect size estimates from the existing literature, which are often provided in the form of metaanalyses, or to use effect sizes generated from pilot studies. In general, these seem like viable and empirically driven ways to determine effect size estimates but recent research suggests that these are problematic due to the same issue: the small samples used to generate the effect sizes (Bakker, van Dijk, \& Wicherts, 2012; Gelman \& Carlin, 2014). A relevant example for infant research is the examination by Bakker and colleagues (2012) of the meta-analysis by McCall and Carriger (1993) that looked at infant habituation to stimuli and cognitive ability. Here Bakker and colleagues demonstrate how the publication biases of publishing only significant results in small samples ( 25) lead to inflated effect sizes. These effect sizes are not robust (see also Simonsohn, Nelson, Simmons, 2014) and result in future research that is based on small samples and incorrect estimate of effect sizes. Similar issues have been noted by Gelman and Carlin (2014) when considering the use of pilot data for setting effect size estimates to determine the power of a study design. Typically, sample sizes are small in pilot studies and can lead to inflated estimates of effect sizes, which ultimately result in an incorrect sample size calculation. 
Given these challenges, we provide some alternative strategies for calculating the effect sizes for infancy and early childhood research? First, using the previous published literature can still give some sense of the range of effect sizes. Also, using studies that have higher sample sizes will give better precision on a likely effect size for the phenomena of interest. Even after completing an exhaustive review of the literature, it is still likely that there will not be studies of adequate sample size to trust that the effect size estimates are correct. At this point you may need to give a set of potential effect sizes that are consistent with the ones from the broader psychological community, which are often as low as .10 and rarely exceed .35 (Bakker, van Dijk, \& Wicherts, 2012). Hence, calculating the sample size needed for the smallest effect of .10 can give you confidence that you have powered your study adequately. Using an open source power calculator like G*Power (http://www.gpower.hhu.de/) can be a useful tool for common developmental psychology analyses such as different $t$ tests, $F$ tests, and Chi-square tests (Faul et al., 2007). Unfortunately, for those working with infants and testing even a simple mean difference between two groups of babies, the sample size needed to find significance at the $p<$ .05 , for a one-tailed $t$-test with .80 power at an effect size of .10 would be approximately 1250 infants per group or 2500 in the full sample. At the higher effect size of .35 at least 100 per group or 200 total infants are needed to be powered. This generally exceeds the sample sizes of most studies in this research area and often what is possible to obtain by any single infant lab. Therefore, innovations in collecting larger samples are an important step for adequately studying very young children.

\section{[Insert Figure 1 Here]}

One potential avenue for increasing sample sizes is to encourage collaborations within or between labs and institutions using the same protocols and analyzing a pooled data set. Figure 1 
illustrates the number of collaborating labs necessary for adequate power across different effect sizes for a one-tailed, independent samples $t$-test under the same assumptions mentioned above ( $p<.05$, with .80 power). As seen in the figure, the larger the effect size, the smaller the number of collaborators. Although 100 collaborators for $d=.10$ seems difficult, there are now multiple archives of data attempting to help researchers easily combine data and share protocols for observing infants. For example, the Dataverse project (https://dataverse.org/) is an open source web application that provides researchers with a space to archive data and receive academic credit for their data. Also, Databrary (Simon, Gordon, Steiger \& Gilmore, 2015) was established to allow for the sharing of video data on infants and young children in hopes of creating more robust data. This has expanded to include voice recordings and also serves as an archive of protocols and coding for researchers to share. Thus, when researchers move to collaborative and more open science for sharing, major strides can be made in researching even the most difficult respondents with robust methods and power to find effects which lead to more replicable science.

\section{Protocol Flexibility}

Another broad area of threats to replicability and rigorous science involves the researcher decisions that are made at all phases of the research process. Many of the practices that occur during a research project fall under a general category of "researcher flexibility" or "researcher degrees of freedom” (researcher DFs; Wicherts et al, 2016), which are the decisions made throughout the conduct of a study that are often not officially noted in a published paper or protocol. This is a critical point where documentation regarding key decisions of the study need to be noted in order for the study to be replicated by others (Asendorpf et al., 2013; Button et al., 2013; Gelman, 2017; Gelman \& Carlin, 2014; Ioannidis, 2005; Poldrack et al., 2017; Schmidt, 
2009; Simmons, Nelson, \& Simonsohn, 2011; Simonsohn, Nelson, \& Simmons, 2014; Steegen, Tuerlinckx, Gelman, \& Vanpaemel, 2016; Wicherts et al., 2016).

As noted by Wicherts et al., 2016, it is fairly common that the decisions made during the collection of data can lead to interviewer bias, incorrectly coded data, change in testing protocol or discarding of data due to stopping rules or outliers. Children can be difficult to test or interview and during these times it may be difficult to stick to the standardized protocols. Therefore, individual researchers may adjust the testing environment to help the child to stay on task or perhaps end the testing session if a child disengages or appears tired or bored. If an interviewer systematically does this, then interviewer bias (non-random errors) enters the data. These types of decisions or adjustments need to be documented in order to understand why there may be differences between findings across labs that are using the same testing protocols. Thus, researchers need to take great care in noting all aspects of a study including all details of the environment in which the data were collected. This is an important decision point and one that directly influences the ability to fully replicate a study.

A recent ethnography by Peterson (2016) specifically highlighted these issues related to the study of infants and young children. For 16 months, Peterson volunteered in three different labs that tested young children. He documented how frequently research assistants would vary from the protocols he was trained to administer and noted this as "protocol flexibility" where a child being fussy or a parent being difficult would lead to test or assessments being administered differently than the standard protocol dictated. These differences were not noted in lab notes or noted in meetings and the data were simply included with other data that were then analyzed for these labs. Even though this study is a small sample design and not generalizable, the research clearly exposed some of the researcher DF issues during the data collection phase of a study. 
Without clear and standard protocols that are rigorously applied across studies and detailed notes about when the researchers has gone "off protocol”, it is unlikely that results can be replicated within studies in the same lab or from independent researchers outside the labs and institutions of the study.

Even though it is often difficult to adhere to protocols when dealing with unexpected situations that occur during data collection, there are some practices that may reduce the flexibility that occurs in infant and early childhood research. For example, providing standard training of the research protocol across the research group helps reduce confusion when collecting data and provides a protocol for appropriately dealing with complex situations the may occur during data collection. When these inevitable deviations do occur in the protocol, then there should be notes of why and when these deviations occurred and the data associate with these differences flagged as being “off-protocol” so that it can be reviewed later. Another useful practice is to consider videotaping all testing sessions so that if there are questions of differences in interviewers or situations, they can be reviewed, coded, documented, and decisions made on the inclusion of the data. In general, ensuring that all decisions, changes, or deviations from protocol are noted during data collection will allow for the assessment of how these differences might influence the findings of the research. Finally, consider creating an online lab notebook (either a simple shared document or potentially using a preregistration template like those available at www.osf.io) where changes in the protocol or decisions made during data collection varied from the intended study. This will make it easier to replicate findings within a research group but also provide documentation for those outside the research group who are using the same protocol. Thus, documenting data collection and procedures can avoid random decisions of data inclusion (or exclusion), can inform methodologies for future studies, and can aid in the 
replicability of findings to determine the robustness of a phenomena for the larger scientific community (see Oakes, 2010).

\section{Analysis Flexibility}

The final major area of concern for reproducible and robust science in infancy research is the data analysis stage. If the study is adequately powered and the research study well executed and documented, then the next phase is to adequately analyze the data. The analyses being used in the study should be driven by the questions or hypotheses. Studies are often a combination of confirmatory (i.e., clear hypotheses that stem from the literature and are being confirmed in the current study) and exploratory analyses (i.e., a set of questions that may be exploratory or unique to the current study and no a priori hypotheses are made regarding the findings). Unfortunately, across the last few decades of research including infancy research, the lines between confirmatory and exploratory analyses have become blurred.

Peterson (2016) noted that researchers were “stacking the deck” in order to find significant findings. He described these researchers as constantly watching the data and looking at computer print-outs to determine whether to continue collecting data or modify what they were doing. These poor research practices fall under the general heading of $p$-hacking, or manipulating the data in ways that try to find significance at the $p<.05$ level (Simonsohn, 2012). p-hacking can involve dropping outliers, running multiple analyses and choosing only the significant finding, or stopping data collection when the data supports the finding the researcher was hoping to find. In most of these cases, the science is confirmatory because there were specific hypotheses being tested and protocols designed to test these hypotheses. But these 
hypotheses and protocols changed across the study making it, at best exploratory, but generally leading to unclear findings.

Another complication and common problem in research exist when the study is being analyzed as designed but the results were either not significant or not as exciting as the researcher or research team had hoped. In this case, additional analyses are conducted, and perhaps other variables added that were not part of the original hypothesis. It is not uncommon, for example, to see interactions as part of a study where only main effects were originally hypothesized. Even though these analyses could be documented as exploratory, the paper is written as if they were part of the original confirmatory hypothesis. In these cases, literature is generally added to the introduction to justify the newly included variables in the study. This type of post-hoc rewriting of the hypothesis has been termed HARKing-hypothesizing after the results are known (Kerr, 1998).

One of the most common issues found related to both HARKing and p-hacking is the multiple analyses that are performed in a study without hypotheses that support them and not corrected for the exploratory nature of the analyses (e.g., Bonferroni Correction; Armstrong, 2014). Often researchers run multiple t-tests looking for group differences or examine correlation matrices looking for significant correlations between variables and then use this information to write-up a study as if they have planned hypotheses for these findings (aka "cherry-picking” the data). When separate hypothesis tests are performed on a large number of variables, the false positive rate is inflated (Gelman \& Loken, 2013). The ability to replicate such a finding that is often found by chance alone is unlikely and the lack of scientific rigor (known as "questionable research practices” or “QRPs”) results in problems in the field in knowing whether or not a true phenomenon has been found (Simmons, Nelson, \& Simonsohn, 2011). 
A common and difficult problem to address is when reviewers ask researchers to add different variables or substantially change analyses in a paper. This is often done to increase the interest or novelty of the research findings. The editors often ask for these changes to be made and the paper is adjusted just as discussed previously, with the introduction rewritten to include the new variables or analyses. In this situation, we have reviewer/editor HARKing and addressing these situations may not be easy or straightforward. One possible way to deal with these cases is to reply with a statement that explains the degree to which changing the analyses in that way would also be changing the question and purpose of the study. Even in this situation, the most straightforward way of reducing HARKing and $p$-hacking is to clearly detail the confirmatory aspects of the questions from the exploratory. This will help reviewers and the larger scientific community to gage the contribution of the research and the next steps needed in the research domain.

\section{Best Practices for Future Research}

Perhaps one of the most important steps to take in increasing the robustness and integrity of the research in infancy and developmental research is to preregister all studies and to clearly delineate confirmatory and exploratory research (Wagenmakers et al., 2012; Chambers \& Munafò, 2013). Preregistration is similar to writing a proposal for a dissertation or grant such that all hypotheses are stated, power of the design is calculated, appropriate sample is selected, measurement is detailed, analyses are pre-planned, and all that is left is to gather the data based on these specifications. Resources are easily available for preregistration. The Open Science Framework (OSF) (https://osf.io/dashboard), for example, is a common website used for uploading preregistered studies. OSF provides researchers with a platform to organize thoughts, timestamp their hypotheses, and even accept feedback from research peers. 
Another avenue that is available is Registered Reports (currently available in Infancy, Infant Behavior and Development, Developmental Science, and British Journal of Developmental Psychology). These differ from preregistered studies (www.cos.io/rr) in that projects are submitted for peer review, revised, and accepted before data collection even begins. If the paper is accepted at this stage, then regardless of strength, direction, or statistical significance in the findings after data collection it will be published in the journal. Use of this type of research practice allows for transparency in the process and increased rigor in the science. Publishing the results of a well-designed study without regard to significant findings also allows for the reduction in publication bias in the literature and for the literature to be corrected as is the general intent of the scientific process.

However, many researchers believe that some of these practices may limit researchers from exploration and discovery—also a hallmark of the scientific process. We would like to bring attention to this point, as the purpose of preregistration and Registered Reports is not to hinder researchers from asking exciting questions and conducting novel research. Instead, the purpose of these scientific practices is to assure that the research is detailed enough so that it is clear how the results of the study were found. The Open Science Collaboration created a uniformed system for researchers to be transparent and collaborative. This aids in replication but also to allow others to evaluate the strength of the research findings and whether it is a topic to continue to pursue.

\section{Conclusion}

The issues of increasing the robustness of infancy and developmental science are important and good scientific practices lead to replicable science that helps to increase the 
integrity of science overall. Even though the psychological "crisis” has been associated with the areas of social and cognitive psychology, it is a crisis that impacts all areas of psychological research because many of the methods we use in experimental and correlational research are similar across psychological science (Tackett et al., 2017). Establishing better practices in infant research, and developmental psychology more broadly, is an important step in making our research more valuable to the community that we seek to help with our research.

We have highlighted some examples of common problems in infancy research though the examples provided are not exhaustive and there are other issues that need to be addressed in developmental research, such as measurement standardization, manipulation checks in experimental research, and sensitivity analysis/robustness checks in correlational research (Duncan, Engel, Claessens, \& Dowsett, 2014). It is hoped that we have provided developmental researchers with some best practices for common issues that occur while conducting research with infants and young children that will increase the robustness and viability of our research.

Ideally, biases would be avoided altogether in research studies if preregistration is used at the beginning of a project. With the preregistration format, researchers provide a specific and comprehensive research plan. If other analyses or results arise, then they should be written as exploratory and researchers should be transparent about their process. Therefore, there is only one confirmatory interpretation of their project in the literature, avoiding the many researcher DFs that exist at the data collection, analysis, or reporting phases. Using an open science practice allows for organization and transparency in the field and is not the only fix but is an important step forward in our science. 


\section{References}

Armstrong, R. A. (2014). When to use the Bonferroni correction. Ophthalmic \& Physiological Optics: The Journal of the British College of Ophthalmic Opticians , 34(5), 502-508. https://doi.org/10.1111/opo.12131

Asendorpf, J. B., Conner, M., De Fruyt, F., De Houwer, J., Denissen, J. J., Fiedler, K., ... \& Perugini, M. (2013). Recommendations for increasing replicability in psychology. European Journal of Personality, 27(2), 108-119. https://doi.org/10.1002/per.1919

Bakker, M. \& Wicherts, J.M. (2011). The (mis)reporting of statistical results in psychology journals. Behavior Research Methods 43 (3) 666-678. https://doi.org/10.3758/s13428011-0089-5

Bakker, M., van Dijk, A., \& Wicherts, J. M. (2012). The rules of the game called psychological science. Perspectives on Psychological Science, 7(6), 543-554. https://doi.org/10.1177/1745691612459060

Button, K. S., Ioannidis, J. P., Mokrysz, C., Nosek, B. A., Flint, J., Robinson, E. S., \& Munafò, M. R. (2013). Power failure: why small sample size undermines the reliability of neuroscience. Nature Reviews Neuroscience, 14(5), 365. doi:10.1038/nrn3475

Chambers, C. D., \& Munafò, M. (2013). Trust in science would be improved by study preregistration. The Guardian, 5.

Duncan, G. J., Engel, M., Claessens, A., \& Dowsett, C. J. (2014). Replication and robustness in developmental research. Developmental Psychology, 50(11), 2417. http://dx.doi.org/10.1037/a0037996 
Faul, F., Erdfelder, E., Lang, A. G., \& Buchner, A. (2007). G* Power 3: A flexible statistical power analysis program for the social, behavioral, and biomedical sciences. Behavior Research methods, 39(2), 175-191.

Gelman, A. (2017). Ethics and Statistics: Honesty and Transparency Are Not Enough. Chance, 30(1), 37-39. https://doi.org/10.1080/09332480.2017.1302720

Gelman, A., \& Carlin, J. (2014). Beyond power calculations: assessing type S (sign) and type M (magnitude) errors. Perspectives on Psychological Science, 9(6), 641-651. https://doi.org/10.1177/1745691614551642

Gelman, A., \& Loken, E. (2013). The garden of forking paths: Why multiple comparisons can be a problem, even when there is no "fishing expedition” or “p-hacking” and the research hypothesis was posited ahead of time. Unpublished Manuscript, Department of Statistics, Columbia University.

Ioannidis, J. P. (2005). Why most published research findings are false. PLoS medicine, 2(8), e124. https://doi.org/10.1371/journal.pmed.0020124

Kerr, N. L. (1998). HARKing: Hypothesizing after the results are known. Personality and Social Psychology Review, 2(3), 196-217. https://doi.org/10.1207/s15327957pspr0203_4

Lindsay, D.S. (2015). Replication in psychological science. Psychological Science 26(12):18271832. https://doi.org/10.1177/0956797615616374

McCall, R. B., \& Carriger, M. S. (1993). A meta- analysis of infant habituation and recognition memory performance as predictors of later IQ. Child development, 64(1), 57-79. https://doi.org/10.1111/j.1467-8624.1993.tb02895.x 
Munafò, M. R., Nosek, B. A., Bishop, D. V., Button, K. S., Chambers, C. D., du Sert, N. P., ... \& Ioannidis, J. P. (2017). A manifesto for reproducible science. Nature Human Behaviour, 1, 0021. doi:10.1038/s41562-016-0021

Oakes, L. M. (2010). Using habituation of looking time to assess mental processes in infancy. Journal of Cognition and Development, 11(3), 255-268. https://doi.org/10.1080/15248371003699977

Open Science Collaboration. (2015). Estimating the reproducibility of psychological science. Science, 349(6251), aac4716. doi: 10.1126/science.aac4716

Peterson, D. (2016). The baby factory: Difficult research objects, disciplinary standards, and the production of statistical significance. Socius, 2, 2378023115625071. https://doi.org/10.1177/2378023115625071

Poldrack, R. A., Baker, C. I., Durnez, J., Gorgolewski, K. J., Matthews, P. M., Munafò, M. R., ... \& Yarkoni, T. (2017). Scanning the horizon: towards transparent and reproducible neuroimaging research. Nature Reviews Neuroscience, 18(2), 115. doi:10.1038/nrn.2016.167

Sawilowsky, S. S. (2009). New effect size rules of thumb. Journal of Modern Applied Statistical Methods, 8(2), 597 - 599.

Schmidt, S. (2009). Shall we really do it again? The powerful concept of replication is neglected in the social sciences. Review of General Psychology, 13(2), 90. http://dx.doi.org/10.1037/a0015108

Simon, D. A., Gordon, A. S., Steiger, L., \& Gilmore, R. O. (2015). Databrary: Enabling sharing and reuse of research video. In Proceedings of the 15th ACM/IEEE-CS Joint Conference on Digital Libraries (pp. 279-280). ACM. 
Simmons, J. P., Nelson, L. D., \& Simonsohn, U. (2011). False-positive psychology: Undisclosed flexibility in data collection and analysis allows presenting anything as significant. Psychological science, 22(11), 1359-1366. https://doi.org/10.1177/0956797611417632

Simonsohn, U. (2012). It does not follow: Evaluating the one-off publication bias critiques by Francis (2012a, 2012b, 2012c, 2012d, 2012e, in press). Perspectives on Psychological Science, 7(6), 597-599. https://doi.org/10.1177/1745691612463399

Simonsohn, U., Nelson, L. D., \& Simmons, J. P. (2014). P-curve: a key to the file-drawer. Journal of Experimental Psychology: General, 143(2), 534. http://dx.doi.org/10.1037/a0033242

Steegen, S., Tuerlinckx, F., Gelman, A., \& Vanpaemel, W. (2016). Increasing transparency through a multiverse analysis. Perspectives on Psychological Science, 11(5), 702-712. https://doi.org/10.1177/1745691616658637

Tackett, J. L., Lilienfeld, S. O., Patrick, C. J., Johnson, S. L., Krueger, R. F., Miller, J. D., ... \& Shrout, P. E. (2017). It’s time to broaden the replicability conversation: Thoughts for and from clinical psychological science. Perspectives on Psychological Science, 12(5), 742756.

Wagenmakers, E. J., Wetzels, R., Borsboom, D., van der Maas, H. L., \& Kievit, R. A. (2012). An agenda for purely confirmatory research. Perspectives on Psychological Science, 7(6), 632-638. https://doi.org/10.1177/1745691612463078

Wicherts, J. M., Veldkamp, C. L., Augusteijn, H. E., Bakker, M., Van Aert, R., \& Van Assen, M. A. (2016). Degrees of freedom in planning, running, analyzing, and reporting psychological studies: A checklist to avoid p-hacking. Frontiers in Psychology, 7, 1832. https://doi.org/10.3389/fpsyg.2016.01832 


\section{Hypothetical Number of Collaborating Labs Needed for Adequate Power} for a one-tailed, independent samples t-test

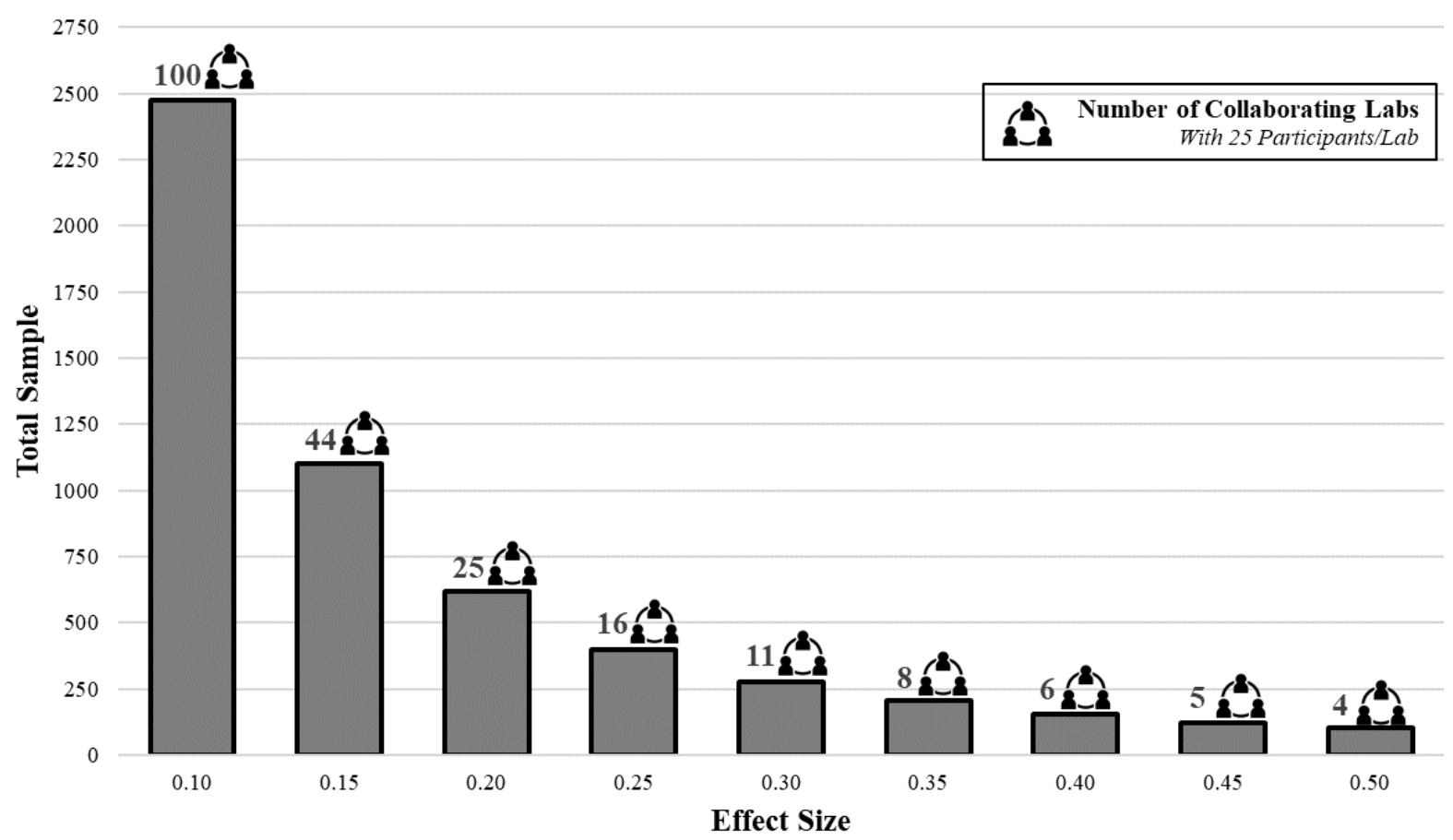

Figure 1. Hypothetical collaborators needed to be adequately powered at the appropriate effect size for a one-tailed, independent samples $t$-test under the assumptions: $p<.05$, powered at .8, with 25 participants per lab. 